placing $\beta \rho^{2}$ by $\frac{1}{2} \beta r_{m}{ }^{* 2}$ with $r_{m}{ }^{*}$ read directly from Fig. 2 of Hillier and Walkley. ${ }^{10}$ Curves (A) and (B) of Fig. 1 show the zero-point energy $E_{1,0} *$ for the reduced cell radius $r_{\max }$ equal to $\rho$ and $r_{m}{ }^{*}$, respectively. Curve (C) is the exact LJ \& D quantum cell model result. ${ }^{4}$ Curve (D) is the square-well data of Hillier and Walkley ${ }^{10}$ using the same $r_{m}{ }^{*}$ as in Curve (B). As pointed out by Feinberg and De Rocco, it comes as no great surprise that the triangle well gives

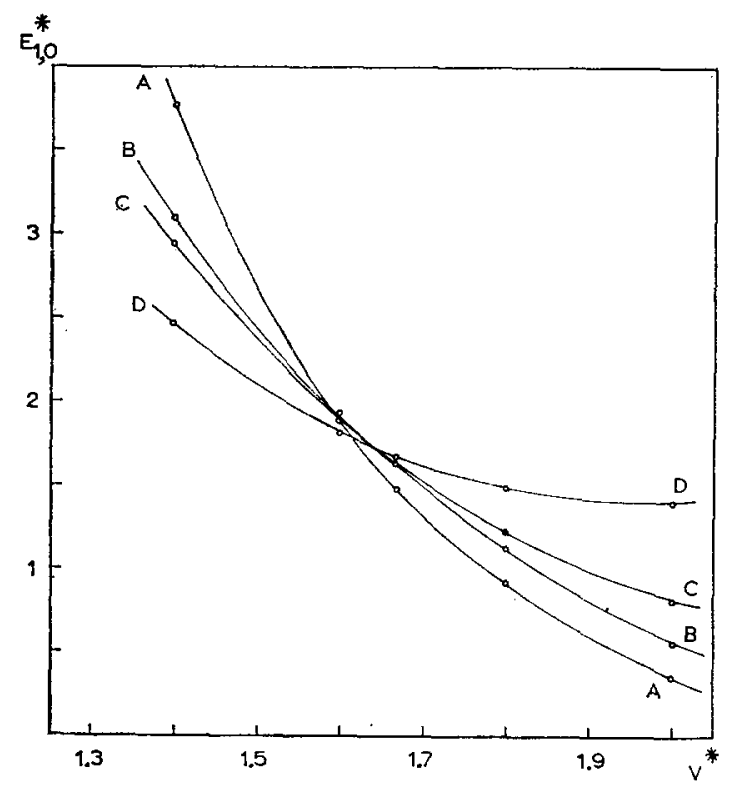

FIG. 1. Reduced zero-point energy for liquid hydrogen as a function of the reduced cell volume. Curve (A) $r_{\max }=\rho$; (B) $r_{\max }=r_{m}{ }^{*}$ calculated by Hillier and Walkley ${ }^{10}$; (C) exact LJ \& D quantum cell values 4,0 ; (D) square-well data of Hillier and Walkley ${ }^{10}$ with effective cell radius $r_{m}{ }^{*}$.

better results than the square well over the range of volumes shown. No attempt has been made to optimize the parameter $n$, which was taken to be 2 throughout the calculations. The results of this exercise indeed lend credence to the intuitive feeling that the triangle well should give reasonable estimates, with minimal computations, for problems which are quite tedious with the LJ \& D potential.

${ }^{1}$ J. E. Lennard-Jones and A. F. Devonshire, Proc. Roy, Soc. (London) A163, 53 (1937).

${ }^{2}$ R. H. Wentorf, Jr., R. J. Buehler, J. O. Hirschfelder, and C. F. Curtiss, J. Chem. Phys. 18, 1484 (1950).

${ }^{3}$ D. Henderson and R. D. Reed, J. Chem. Phys. 40, 975 (1964)

4 R. D. Reed and D. Henderson, J. Chem. Phys. 41, 2705 (1964).

${ }^{5}$ M. J. Feinberg and A. G. De Rocco, J. Chem. Phys. 41, 3439 (1964).

${ }^{6}$ F. C. Auluck, Proc. Natl. Inst. Sci. India 7, 133 (1941); and 8, 147 (1942).

7 S. Chandrasekhar, Astrophys. J. 97, 268 (1943)

${ }^{8} \mathrm{~L}$. J. Slater, Confuent Hypergeometirc Functions (Cambridge University Press, Cambridge, England, 1960).

9 R. P. Hurst and J. M. H. Levelt, J. Chem. Phys. 34, 54 (1961).

${ }^{10} \mathrm{I}$. H. Hillier and J. Walkley, J. Chem. Phys. 41, 3205 (1964).

\section{Determination of the Phonon Spectrum of Polyethylene by Neutron Scattering*}

\author{
G. C. SUMMERFIELD \\ Department of Nuclear Engineering, The University of Michigan, \\ Ann Arbor, Michigan
}

(Received 15 March 1965)

QEVERAL recent papers $^{1-3}$ have reported the measurement of the low-energy part of the frequency spectrum of polyethylene by inelastic scattering of thermal and cold neutrons. The interpretation of these experiments was based on the following expression for the neutron cross section

$$
\begin{array}{r}
\frac{d^{2} \sigma}{d \Omega d \epsilon}=\frac{\sigma_{0} N k^{\prime}}{\pi k} e^{-2 W}\left\{\delta(\epsilon)+\frac{\hbar^{2} K^{2}}{2 m} \int_{0}^{\infty} d \epsilon^{\prime} \frac{g\left(\epsilon^{\prime}\right)}{N} \frac{m}{3 \epsilon^{\prime} M}\right. \\
\left.\times\left[\frac{\delta\left(\epsilon^{\prime}-\epsilon\right)}{1-e^{-\beta \epsilon^{\prime}}}+\frac{\delta\left(\epsilon^{\prime}+\epsilon\right)}{e^{\beta \epsilon^{\prime}}-1}\right]\right\},
\end{array}
$$

where $\sigma_{0}$ is the bound-atom neutron cross section for hydrogen, $\hbar \mathbf{k}$ and $\hbar \mathbf{k}^{\prime}$ are the initial and final neutron momenta, $\mathrm{H}=\mathbf{k}-\mathbf{k}^{\prime}, \epsilon=\hbar^{2}\left(k^{2}-k^{\prime 2}\right) / 2 m, m$ is the neutron mass, $M$ is the mass of $\mathrm{C}_{2} \mathrm{H}_{4}, N$ is the number of $\mathrm{C}_{2} \mathrm{H}_{4}$ groups in the extended chain, $g(\epsilon)$ is the phonon frequency distribution, and $e^{-2 W}$ is the Debye-Waller factor.

If the neutron cross section is given by Eq. (1), it is a simple matter to extract $g(\epsilon)$ from a neutronscattering experiment. However, Eq. (1) contains several approximations that do not seem to be adequate for polyethylene.

We begin our discussion by detailing those assumptions that seem to apply for polyethylene, and we note the extra approximations that must be made to derive Eq. (1).

The static neutron cross section for hydrogen is significantly larger than that for carbon, and the hydrogen cross section is almost entirely incoherent. Thus, we consider incoherent scattering from the hydrogen atoms. Our model for polyethylene follows that of Tasumi et $a l^{4}$ and Lin and Koenig, ${ }^{5}$ that is, isolated, long, transplanar chains with random orientation. Since the polyethylene targets are highly crystalline ( $290 \%)$, this approximation appears to be quite good. It should be noted that the results of Tasumi ${ }^{4}$ and Miyazawa and Kitagawa ${ }^{6}$ indicate that intermolecular forces will introduce some structure in the low-energy part of the scattered neutron spectrum. It is believed that this structure has been observed in neutron experiments.

With these approximations and assuming only harmonic forces in the molecule, the neutron cross section is ${ }^{7}$

$$
\frac{d^{2} \sigma}{d \Omega d \epsilon}=\frac{N k^{\prime}}{2 \pi^{2} \hbar k} \sigma_{0}\left\langle e^{-Z(0)} \int_{-\infty}^{\infty} d t e^{-8 e t / \hbar} e^{Z(t)}\right\rangle,
$$


where

$$
\begin{aligned}
Z(t)=\sum_{j q \alpha \alpha^{\prime}} \frac{\hbar^{2} K_{\alpha} K_{\alpha^{\prime}}}{2 m} \gamma_{j}{ }^{\alpha}(q) \gamma_{j^{\alpha^{\prime}}}(q) \frac{1}{N \hbar \omega_{j}(q)} \\
\quad \times\left[\frac{\exp \left[-i \omega_{j}(q) t\right]}{1-\exp \left[-\beta \hbar \omega_{j}(q)\right]}+\frac{\exp \left[-i \omega_{j}(q) t\right]}{\exp \left[\beta \hbar \omega_{j}(q)\right]-1}\right] ;
\end{aligned}
$$

$\omega_{j}(q)$ is the phonon energy for the $j$ branch, $\gamma_{j}{ }^{\alpha}(q)$ is the $\alpha$ component of the polarization vector for a hydrogen atom, and the brackets \langle\rangle indicate the average over the orientations of the molecule.

The one-phonon approximation is obtained by using $e^{Z(t)} \approx 1+Z(t)$ in Eq. (2). ${ }^{8}$ Then, we define the distribution $G_{j}^{\alpha \alpha^{\prime}}(\epsilon)$ by

$$
\sum_{q} \gamma_{j}^{\alpha}(q) \gamma_{j}^{\alpha^{\prime}}(q) F\left(\hbar \omega_{j}(q)\right)=\int_{0}^{\infty} d \epsilon^{\prime} G_{j}^{\alpha \alpha^{\prime}}\left(\epsilon^{\prime}\right) F\left(\epsilon^{\prime}\right)
$$

We should note that to obtain a nonzero cross section we must have $G_{j}^{\alpha \alpha^{\prime}}(\epsilon)$ approach zero at least as fast as $\epsilon^{2}$ for $\epsilon \rightarrow 0$. This behavior does not agree with the results of Refs. 4 and 5, but it does agree with the more realistic calculation of Ref. 6 . Then we have

$$
\begin{aligned}
& \frac{d^{2} \sigma}{d \Omega d \epsilon}=\frac{\sigma_{0} N k^{\prime}}{\pi k}\left\langle e^{-Z(0)} \delta(\epsilon)\right.+e^{-Z(0)} \sum_{j \alpha \alpha^{\prime}} \frac{\hbar^{2} K_{\alpha} K_{\alpha^{\prime}}}{2 m} \int_{0}^{\infty} d \epsilon^{\prime} \frac{G_{j}^{\alpha \alpha^{\prime}}\left(\epsilon^{\prime}\right)}{N \epsilon^{\prime}} \\
&\left.\times\left[\frac{\delta\left(\epsilon^{\prime}-\epsilon\right)}{1-e^{-\beta \epsilon^{\prime}}}+\frac{\delta\left(\epsilon+\epsilon^{\prime}\right)}{e^{\beta \epsilon^{\prime}}-1}\right]\right\rangle .
\end{aligned}
$$

In order to bring Eq. (3) into the form of Eq. (1), we must perform the average over orientations by replacing $K_{\alpha} K_{\alpha^{\prime}}$ by its average over orientation $\delta_{\alpha \alpha^{\prime}} K^{2} / 3$, and we must take $\sum_{\alpha}\left[\gamma_{j}{ }^{\alpha}(q)\right]^{2}$ equal to $m / M$. A detailed study of these approximations would require a determination of the $\gamma_{j}{ }^{\alpha}(q)$. Considering these approximations, it is not meaningful to demand much more than qualitative agreement between the computations of Refs. 4, 5, and 6 and the results of neutron experiments using Eq. (1). Calculations of the polarization vectors are in progress and these results used in Eq. (3) should be in quantitative agreement with experiments, thus providing considerable detailed information about the polyethylene molecule, including such factors as intermolecular and anharmonic forces.

In conclusion, let us note that, while the expected qualitative agreement between theory and experiment is obtained using $\mathbf{E q}$. (1), the measured frequency distribution above $0.025 \mathrm{eV}$ appears to be consistently higher than the theoretical distribution. This discrepancy can be eliminated by a slight modification of the approximation used for $\gamma_{j}^{\alpha}(q)$. In particular, taking $\sum_{\alpha}\left[\gamma_{\nu_{5}}{ }^{\alpha}(q)\right]^{2}=m / M$ and $\sum_{\alpha}\left[\gamma_{\nu_{\theta}}{ }^{\alpha}(q)\right]^{2}=m / 2 M$ brings theory and experiment into better agreement. ${ }^{\circ}$

* Work supported by the U. S. Atomic Energy Commission.

1 H. Danner et al., J. Chem. Phys. 40, 1417 (1964).

$2 \mathrm{H}$. Boutin et al., Proceedings of the IAEA Conference on the Inelastic Scattering of Neutrons, Bombay, India (to be published).
${ }^{3}$ W. Myers, J. Donovan, and J. S. King, J. Chem. Phys. 42, 4299 (1965).

1M. Tasumi et al., J. Mol. Spectry. 9, 261 (1962); and 11, 422 (1963); and M. Tasumi, thesis, University of Tokyo, 1964.

${ }^{5}$ T. P. Lin and J. L. Koenig, J. Mol. Spectry. 9, 228 (1962).

6 T. Miyazawa and T. Kitagawa, Polymer Letters 2, 395 (1964).

${ }^{7}$ C. Zemach and R. Glauber, Phys. Rev. 101, 118 (1956).

8 One should always compute the two-phonon term arising from $\frac{1}{2}[Z(t)]^{2}$ to ensure that the one-phonon approximation is valid. In the work described below this check is always made.

$\checkmark$ The notation $\nu_{5}$ and $\nu_{9}$ is that of Ref. 4 .

\section{Electron Spin Resonance of ${ }^{15} \mathrm{~N}$ Atoms}

\author{
CASPer J. Ultee \\ Research Laboratories, United Aircraft Corporation \\ East Hartford, Connecticut
}

(Received 2 April 1965)

$\mathbf{T}$ HE electron spin resonance spectrum of gaseous ${ }^{14} \mathrm{~N}\left({ }^{4} S\right)$ has been observed by several authors. ${ }^{1-3}$ The spectrum consists of three lines with a $g$ value of 2.002 and a splitting of $3.8 \mathrm{G}$. Recently, two additional lines were discovered in the gas-phase atomic-nitrogen spectrum (Fig. 1). The spectra were observed with a Varian V-4502 spectrometer using $100-\mathrm{kc} / \mathrm{sec}$ modulation and a V-4531 multipurpose cavity. This cavity operates in the TE-102 mode and accommodates sample tubes up to $11-\mathrm{mm}$ diam. The nitrogen was dissociated with a $2450-\mathrm{Mc} / \mathrm{sec}$ discharge in a flow system and pumped through the spectrometer cavity. The flow system is constructed from 11-mm-o.d. quartz tubing; the discharge is located about $1 \mathrm{~m}$ from the spectrometer cavity. The nitrogen used was Linde "H. P. Dry," and no wall poisons were used in the flow system. Typical operating conditions were $10-\mathrm{mm} \mathrm{Hg}$ pressure and flow rates of 100 to $150 \mathrm{~cm}^{3}$ (NTP)/ $/ \mathrm{sec}$.

The additional lines are explained as arising from ground-state ${ }^{15} \mathrm{~N}$ atoms. The first-order strong-field energies for $\mathrm{N}$ are given by ${ }^{1} W\left(M_{I} M_{J}\right)=A M_{I} M_{J}+$ $M_{J} g_{J} \mu_{0} H+M_{I} g_{T} \mu_{0} H$. For nitrogen atoms with a $1 s^{2} 2 s^{2}\left({ }^{2} P\right)^{3}\left({ }^{4} S_{3}\right)$ configuration, the hyperfine coupling constant $A$ would be zero. However, when configura-

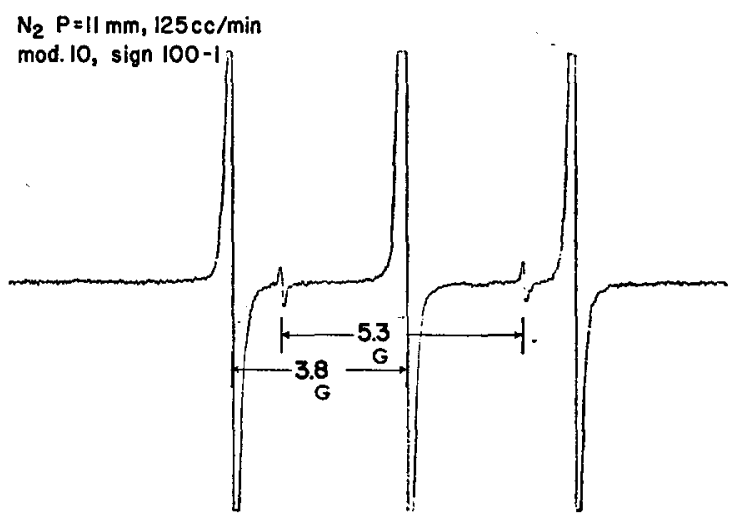

FIG. 1. ESR spectrum of atomic nitrogen. 